\title{
Next Generation Networks and Services in Slovenia
}

\author{
Andrej Kos, Janez Bešter, and Peter Homan \\ University of Ljubljana, Faculty of Electrical Engineering, Laboratory of \\ Telecommunications, Tržaška 25, 1000 Ljubljana, Slovenia, \\ \{andrej.kos, janez.bester, peter.homan\}@fe.uni-lj.si \\ http://www.ltfe.org
}

\begin{abstract}
This paper provides an overview of development of telecommunications in Slovenia. Major systems, networks and services are briefly considered. The combination of own generic research and critical mass of knowledge had and still has a very positive influence the on development of telecommunications in Slovenia. We propose a two-level network architecture consisting of a simplified data forwarding plane and service control plane. Future technological development and the proposed role of Slovenia as a regional telecommunications hub are presented.
\end{abstract}

\section{Introduction}

Recent years have been marked with significant advances in telecommunications. The main reasons for fast development are:

1. Fast development of new technologies

2. Rapidly falling prices of networking equipment and bandwidth

3. Rapidly falling prices of services

4. Changing the basic platform of telecommunications from connection oriented networks to connectionless, packet-based networks

5. Convergence

6. Rapid shift of importance from technology towards services

7. Deregulation and liberalization

In 2000 there were still some doubts about the general development path of telecommunications. Two scenarios were possible: evolution and revolution. Revolutionary scenario anticipated the advent of new, small, specialized, and technically very advanced actors. The services would all be provided over IP infrastructure. Evolutionary scenario anticipated gradual transformation of classical telecommunications in 10 to 15 years from PSTN/ISDN-centric to IP-centric companies. It is now clear that the future development in telecommunications will follow evolutionary path. Telecoms, on contrary to new players, typically have large investments in embedded base and strong revenue-generating existing services (voice) that help fund extensive and expensive network as well as service upgrades. Areas where investment is particularly intense are mobile, broadband, and Internet.

E. Gregori et al. (Eds.): NETWORKING 2002, LNCS 2345, pp. 1147-1152 2002.

(C) Springer-Verlag Berlin Heidelberg 2002 


\section{State of Telecommunications in Slovenia}

Slovenia has relatively well developed telecommunications sector. Some important characteristics of Slovenian telecommunications are summarized in Table 1

Table 1. Main telecommunications indicators

\begin{tabular}{lr}
\hline Indicators & End of 2001 \\
\hline Population & 1.971 .000 \\
GDP/inhabitant & $€ 10.840$ \\
ISDN/PSTN density & $45 \%$ \\
Mobile density & $70 \%$ \\
Internet users & $35 \%$ \\
CaTV penetration (households) & $37,5 \%$ \\
Digitalization & $99 \%$ \\
\hline
\end{tabular}

Slovenia belongs among 15 countries in the world that have generic telecommunications development and are capable of developing, producing and exporting advanced telecommunications systems and solutions. There is tight cooperation between industry and academic institutions. Combination of own generic research and critical mass of knowledge had and still has very positive influences on development of telecommunications in Slovenia.

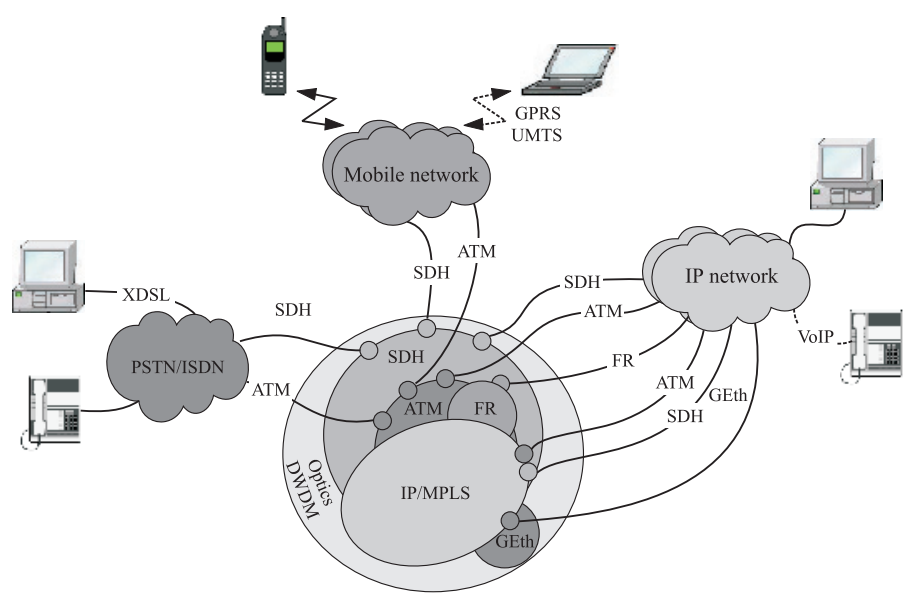

Fig. 1. Core infrastructure

Core infrastructure that supports all three main segments; fixed telephony, mobile, and IP is shown in Fig. 1, It is based on optical cable systems upgraded 
with different technologies on different layers, such as DWDM, SDH, FR, ATM, Gigabit Ethernet, MPLS, and IP. It is mainly provided by Telekom Slovenije. In lesser extent it is also provided by Elekto-Slovenija, Slovenian Railways, and Motorway Company in the Republic of Slovenia. The latter offer leased line services over SDH infrastructure. Fixed telephone network is currently still the most important Slovenian telecommunications infrastructure. At the end of 2000 digitalization rate reached $99 \%$ and the PSTN/ISDN penetration is $45 \%$. The penetration of ISDN and centrex together is $7.3 \%$. Fixed telephone network is structured in two-level hierarchy; primary (PX) and secondary (SX), which is hierarchically higher than the primary. Broadband ADSL services over copper access network are available from the beginning of 2001.

Mobile communications are well developed with one of the highest penetration rates in Europe. Three mobile operators, Mobitel, Si.mobil, and Western Wireless International are operating in Slovenia. Service provider Debitel uses Mobitel's GSM network. At the end of 2001 the penetration rate of mobile users was over $70 \%$. Comparison of mobile penetration rates with other European countries is shown in Fig. 2. The data is valid for September 2001.

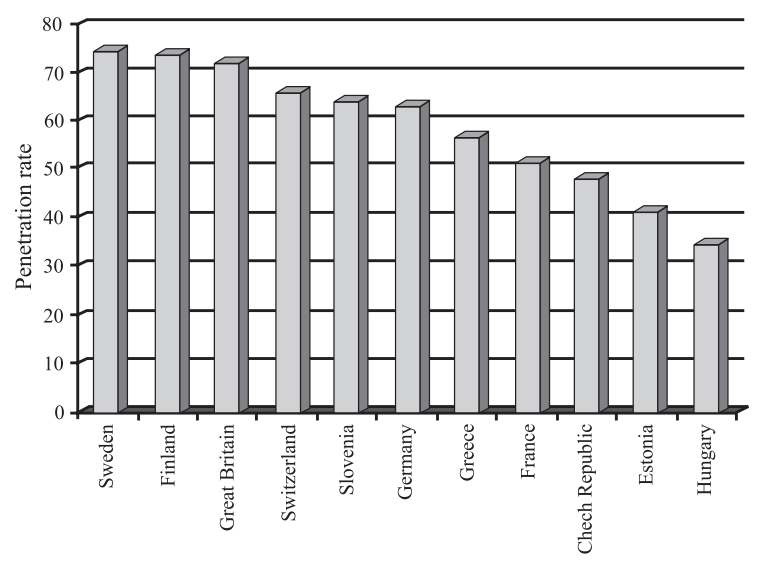

Fig. 2. Comparison of mobile penetration rates (September 2001)

As in the rest of the Europe, the number of people using the Internet continues to grow. In October 2001 there were some 700.000 (35\%) Internet users. A user for the above figure is defined as someone who has used the Internet at least once in the past three months. Of these 700.000 users,

- 500.000 use the Internet at least once per month

- 400.000 use the Internet at least once per week

- 300.000 use the internet on a daily basis

The biggest internet service provider in Slovenia is Siol. It manages the biggest core commercial network and currently offers dial-up access, leased lines, ADSL, 
and Ethernet access. In addition to different types of access to the Internet, Siol offers services such as VPNs, web hosting, all standard IP services, and many new application services, such as audio/video, e-commerce, and distance learning. The other big player in the field of Internet is Academic and Research Network of Slovenia. The main task of Arnes is development, operation and management of the communication and information network for education and research. There is a variety of smaller commercial ISPs that provide internet services, such as access to the Internet, web hosting, consulting and similar. Currently there are more than 100 CaTV operators in Slovenia, which provide services to around 250.000 Slovenian households and 750.000 users respectively. Thus the CaTV penetration rate is $37.5 \%$. In some urban areas the penetration rate is more than $90 \%$. However the great majority of operators are small companies owned by local communities.

\section{Convergence}

As shown in Fig. 11 telecommunications today are based on three pillars: fixed, mobile and IP. Technologically all three can support voice and data/internet. Up to now terminals for fixed telephone network were classical telephone terminals. With the advent of xDSL, the access telephone network is being used for broadband data as well. GSM mobile networks were primarily built to support voice, but with HSCSD, GPRS and UMTS more and more data traffic will be transported over mobile networks. In the past typical usage of IP networks was data, but with the advent of VoIP, IP networks are being used for voice as well. Especially it is expected that the boundary between mobile operators and Internet service providers will blur due to strong cross-area expansion. With the advent of ADSL there is also a similar blurring of the boundary between fixed operators and Internet Service providers.

General convergence trends that can be identified are:

1. Voice is migrating from fixed to mobile networks (overall voice is growing, whereas there is a decline in fixed voice)

2. Fixed networks will be used for broadband data

3. IP networks are converging into a common infrastructure for all existing and new services through implementation of MPLS

\section{Future Development}

Till the end of 2000 the core network was working mainly in a connection oriented transport fashion, Anticipated technological evolution of core network in general is presented in [12], where it should be noted that although today's vision of next generation core network is IP/MPLS/GMPLS over DWDM, existing, proven and well-known technologies such as SDH, ATM and Gigabit Ethernet would still be used for a long time. As discussed below only their role might be slightly different. 


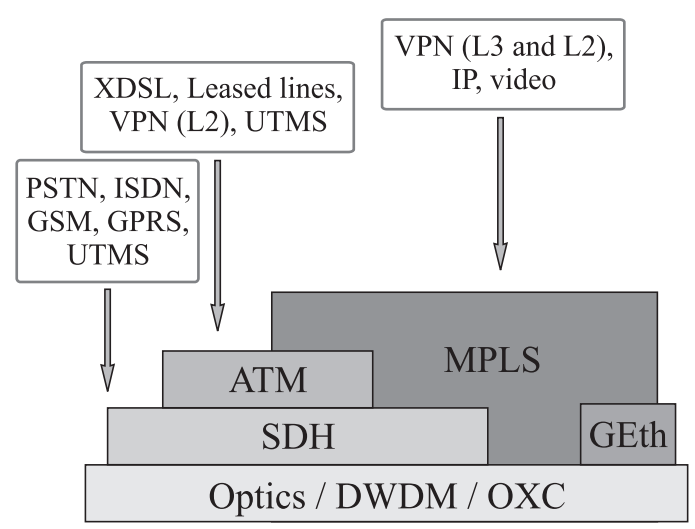

Fig. 3. Concept of contemporary network architecture and its main usage

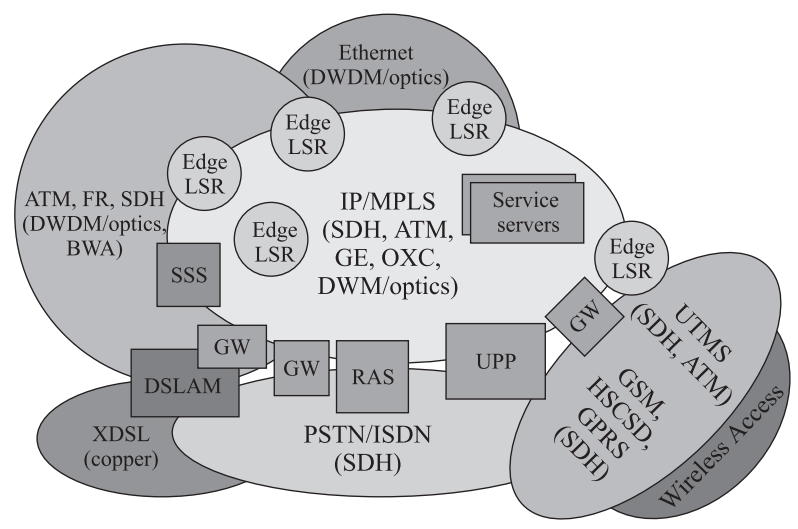

Fig. 4. Next generation network

According to general evolution of core network the concept of future network architecture and its main usage will change as shown in Fig. 3. The concept is based on the following facts:

1. IP protocol has become the convergence layer for majority of services

2. MPLS and its generalization GMPLS have become the core technology of choice that in addition to connection oriented approach support many new functionalities in terms of routing, signaling, control and QoS support

3. ATM as a layer 2 technology is with ADSL and ATM switches at customer sites migrating towards access

4. Voice services will still for some time be accessed via classical terminals, mainly mobile. VoIP functionality will be through media gateways first introduced mainly in the core as voice trunking

In [3] framework for next generation network is proposed. Logically it is a two-level network architecture, which consists of service control layer 
and transport layer. The transport is service independent. We propose next generation network, of which technology aware view is shown in Fig. 44 45 (extended version of this paper can be found on http://www.Itfe.org/pdf/networking2002_extended.pdf). Most of the intelligence is in edge devices. Edge devices' functionalities include termination of different access technologies, data format adaptation for transport over core network, service gateways, such as QoS mappings, connection admission control, classification, metering, marking, dropping, authorization, accounting, fire-walling, address translation, security, and others.

\section{Conclusion}

In the article the overview of development in the field of telecommunications in Slovenia is presented. The combination of own generic research and critical mass of knowledge had and still has very positive influences on development of telecommunications. We propose a two-level network architecture consisting of a simplified data forwarding plane and service control plane. Service control plane is mostly implemented in edge devices, in the form of different gateways and servers. Slovenia with less than 2 million inhabitants is relatively small market and will in global markets have to find its place in niche segments. With a lot of technological know-how, unique geographic position, a lot experience, and good relationships with all neighboring countries, one among most important niche segments is being a telecommunications hub.

Acknowledgements. The authors would like to thank Matej Eljon and the anonymous reviewers for their thoughtful review and useful suggestions.

\section{References}

1. Kos, A., Bešter, J.: Role of MPLS in Modern Telecommunications Networks. International Symposium Viable Telecommunications VITEL 2000, Technologies and Communication for the Online Society, Ljubljana, Slovenia (2000) C45-C49

2. Banerjee, A., et al.: Generalized Multiprotocol Label Switching: An overview of Routing and Management Enhancements. IEEE Commun. Mag., vol. 39, no. 1 (2001) 144-150

3. Moridera, A., Murano, K., Mochida, Y.: The Network Paradigm of the $21^{\text {st }}$ Century and Its Key Technologies, IEEE Commun. Mag., vol. 38, no. 11 (2000) 94-98

4. Rockström, A.: Technology as a Driver for New Business Logic. IEEE Commun. Mag., vol. 38, no. 11, (2000), 100-104

5. Žurbi, R.: Signalling and Control Protocols in Next Generation Networks. M.Sc. Thesis, Faculty of Electrical Enginering, University of Ljubljana, Ljubljana, Slovenia (2001) 